\title{
I flussi migratori: questioni controverse che attraversano la cultura e il diritto della società contemporanea
}

\author{
Roberta Bisi
}

\section{Riassunto}

I flussi migratori rappresentano un fattore di trasformazione della società, dell'economia, dei servizi e della cultura che pongono rilevanti interrogativi a tutti i settori della comunità, evidenziandone spesso le contraddizioni, e alimentando perplessità anche per quanto concerne il concetto di identità, inteso come apertura e continuo rimando ad altro da sé.

\section{Résumé}

Les flux migratoires représentent un facteur de transformation de la société, de l'économie, des services et de la culture. C'est pourquoi, ils posent une série de questions considérables à tous les secteurs de la communauté, mettant souvent en évidence les contradictions et soulevant des incertitudes aussi sur le concept d'identité. Ce dernier doit être vu comme une ouverture et un renvoi continu à autrui.

\section{Abstract}

Migration flows are gradually transforming the culture, the services and the economy of our society highlighting a series of contradictions and uncertainties involving the concept of identity which needs to be regarded and reassessed in the light of the new perspectives developed by the modified social situations.

Key words: migration flows; culture; law; identity; citizenship.

\footnotetext{
- Professore ordinario (settore scientifico-disciplinare SPS/12 - sociologia giuridica, della devianza e mutamento
} sociale) presso la Scuola di Scienze Politiche - Università di Bologna. 
Questo Interlabo, organizzato dal Centro interdisciplinare di ricerca sulla vittimologia e sulla sicurezza dell'Università di Bologna (C.I.R.Vi.S.) e dal Groupe Europeén de Recherches sur les Normativités (Gern) rappresenta un'ottima occasione per formulare alcune riflessioni su un tema di grande attualità: i flussi migratori e, a questo proposito, vorrei riferirmi, partendo da uno dei brani più noti e citati dell'antropologia italiana, al ricordo dell'etnologo Ernesto de Martino quando racconta di una volta in Calabria dove, cercando una strada, egli e i suoi collaboratori fecero salire in auto un anziano pastore perché indicasse loro la giusta direzione da seguire, promettendogli di riportarlo poi al posto di partenza. L'uomo salì in auto pieno di diffidenza, che si trasformò via via in una vera e propria angoscia territoriale, non appena dalla visuale del finestrino sparì alla vista il campanile di Marcellinara, il suo paese. Il campanile rappresentava per l'uomo il punto di riferimento del suo circoscritto spazio domestico, senza il quale egli si sentiva realmente spaesato. Quando lo riportarono indietro, in fretta, l'uomo stava penosamente sporto fuori dal finestrino, scrutando l'orizzonte per veder riapparire il campanile. Solo quando lo rivide, il suo viso finalmente si riappacificò ${ }^{1}$.

Un brano questo che contribuisce a mettere a fuoco il problema delle "patrie culturali", di luoghi che acquisiscono un valore psichico, divenendo l'indice soggettivo del rapporto tra l'essere umano e lo spazio circostante, che raccoglie al suo interno gli aspetti interiori,

${ }^{1}$ E. de Martino, La fine del mondo. Contributo all'analisi delle apocalissi culturali, Einaudi, Torino, 1977; D. Altobelli, "I luoghi della tipicità e della tradizione”, in M. Ruini (a cura di), Dentro e fuori la città, Edizioni Nuova cultura, Roma, 2010, pp. 77-97. intimamente significativi, e quelli collettivi, storicamente determinati: in altri termini, dall'anima, dalle forme archetipiche alla realtà dei luoghi e degli ambiti della vita quotidiana ${ }^{2}$.

Il luogo è un'entità raccolta che, a volte, può essere chiusa, semiaperta od aperta e che rimanda al senso del riparo e del ritorno. E' vero comunque che, come sovente accade ai migranti, il luogo si può alterare e può perdere quelle caratteristiche di protezione e di accoglienza che gli erano in precedenza attribuite ${ }^{3}$ Negli interventi previsti nel corso della giornata di studio si è fatto riferimento ad un caso di omicidio volontario aggravato, avvenuto nel 2010 in provincia di Modena, commesso da padre e figlio, entrambi pakistani, nei confronti della rispettiva moglie e madre, colpevole di aver condiviso il rifiuto opposto dalla figlia alla volontà del padre di darla in sposa ad un uomo figlio di un suo fratello e persona di suo gradimento. La figlia, intervenuta per soccorrere la madre, veniva colpita e riportava gravissime lesioni personali, con riserva di prognosi e necessità di intervento chirurgico d'urgenza.

La ragazza, secondo le testimonianze raccolte da alcune compagne di scuola, aveva confidato loro di essere stata promessa sposa dal padre e sebbene inizialmente, con l'aiuto ed il supporto materno, avesse rifiutato, successivamente, a seguito dei maltrattamenti ai quali veniva costantemente sottoposta la madre, ella aveva accettato di sposare un suo cugino. L'inserimento della ragazza nei servizi educativi e

2 E. Liotta, Su anima e terra. Il valore psichico del luogo, Magi, Roma, 2005.

3 R. Bisi, "Quale spazio per la vittima nella società contemporanea?", in A.Balloni (a cura di), Cittadinanza responsabile e tutela della vittima, Clueb, Bologna, 2006, pp. 61-72. 
nella scuola del Paese di immigrazione ha profondamente inciso nei legami tra le generazioni ed anche nella comunicazione in ambito familiare tanto che la ragazza, per meglio e più velocemente apprendere la lingua italiana, si rivolgeva ad un'insegnante privata che le aveva offerto tale possibilità. In tal modo la ragazza, come avviene sovente in queste situazioni, si avventurava da sola in un mondo di nuove parole e di nuove conoscenze lasciando "i genitori sull'altra riva"4, compromettendo pertanto l'autorità e il prestigio genitoriale.

Dalle testimonianze raccolte si evince che la donna uccisa aveva da tempo chiesto di poter essere allontanata dall'abitazione coniugale e allorquando i carabinieri la convinsero a rientrare nella propria abitazione, dopo aver parlato con il marito, raccomandandole di presentarsi il giorno successivo in caserma per sporgere denuncia, la figlia, di fronte a questo invito, disse: "se ci sarà ancora".

Il medico di base, inoltre, sentita dopo l'omicidio commesso, riferì ai carabinieri che da circa un anno le figlie le avevano comunicato di vivere in un clima familiare di tensione, fatto di minacce e di privazioni.

Da questo punto di vista, è evidente come l'immigrazione rappresenti un fattore di trasformazione della società, dell'economia, dei servizi e della cultura che pone rilevanti interrogativi a tutti i settori della comunità, evidenziandone spesso le contraddizioni. Sovente, l'atteggiamento occidentale sembra voler confinare i delitti d'onore, intrinsecamente legati alle pratiche di segregazione di donne e bambine costrette a matrimoni forzati,

4 R. Beneduce, Frontiere dell'identità e della memoria, nell'ambito della cultura patriarcale presente in molti Paesi a maggioranza musulmana, e al contempo a giustificarli per rispettare le differenze culturali ${ }^{5}$. In tal senso, alcuni anni fa (2007), due casi di cronaca giudiziaria misero ben in evidenza il rapporto problematico tra multiculturalismo e diritti fondamentali: un uomo, di origine sarda e migrato in Germania da alcuni anni, venne condannato da un Tribunale tedesco (Bückeburg) per aver stuprato e seviziato la propria fidanzata. Lo sdegno e lo stupore nei confronti della condanna erano legati al fatto che il Tribunale tedesco aveva ritenuto di concedere al condannato una riduzione di pena poiché la sua condotta sarebbe stata influenzata dalle "particolari impronte culturali ed etniche". Il secondo caso riguarda un immigrato pakistano condannato dal Tribunale di Brescia per l'omicidio della figlia con l'aggravante di aver

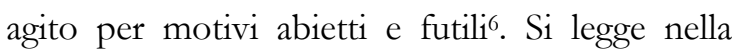
sentenza della Cassazione penale, Sezione I, 18 febbraio 2010, n.6587: “ il motivo è abietto le volte in cui la motivazione dell'agente ripugni al comune sentire della collettività; ed è altresì vero che nella valutazione di siffatto rapporto di 'repulsione' il Giudice di merito non possa prescindere, nel suo scrutinio, dalle ragioni soggettive dell'agire in termini di riferimenti culturali, nazionali, religiosi della motivazione dell'atto criminoso; ma nella specie, [...] la motivazione assorbente dell'agire dell'imputato è scaturita da un patologico e distorto rapporto di

FrancoAngeli, Milano, 1988.

${ }^{5}$ L. Welchman, Honour and Violence Against Women in a Modern Shar'I Discourse (disponibile sul sito: http://eprints.soas.ac.uk/7665/1/Welchman_in_HA WWA_2007_pre-publication.pdf).

6 P. Parolari, "Reati culturalmente motivati. Una nuova sfida del multiculturalismo ai diritti fondamentali”, Ragion pratica, 31, 2008, pp. 529-558. 
'possesso parentale', essendosi la riprovazione furiosa del comportamento negativo della propria figlia fondata non già su ragioni o consuetudini religiose e culturali [...] bensì sulla rabbia per la sottrazione al proprio reiterato divieto paterno".

Il movente dell'omicidio sarebbe stato dettato dalla necessità di ristabilire l'onore della famiglia, oltraggiato dal comportamento della figlia che aveva scelto uno stile di vita occidentale. Questi due casi, pur nella loro diversità, si trovano accomunati nella relazione, assai controversa, tra identità culturale e condotta penalmente rilevante degli imputati. In particolare, il fattore culturale ha giocato pro reo nel primo caso, mentre nel caso del pakistano ha pesato contra reum. Sembra, pertanto, "normale" discutere di attenuante culturale o anche di aggravante nei casi di delitti d'onore commessi da maschi musulmani e, d'altro canto, vengono criminalizzati altri comportamenti quali, ad esempio, l'uso del velo ${ }^{7}$.

E' vero che le migrazioni, come in precedenza anticipato, rappresentando un fattore di trasformazione della società, pongono molti interrogativi anche per quanto concerne il concetto di identità che trova la sua piena realizzazione sul piano delle pratiche di vita attraverso le quali l'uomo si mette in relazione con il mondo mediante il suo conoscere e il suo agire. Identità quindi non come chiusura, bensì come apertura, un continuo rinvio ad altro da sé.

Di qui l'indispensabile riconoscimento del vincolo fra mutamento e senso di una cultura

\footnotetext{
7 S. Ceccanti, "Se l'Italia si 'difende' dal burqa e dimentica la cittadinanza"; M. Nussbaum, "Proibire il velo significa discriminare", Reset, 134, 2012 (disponibile al sito: http://www.reset.it/magazine/134).
}

che ha accumulato la sua esperienza e le sue motivazioni nel tempo. Nel momento in cui la realtà locale avverte che tale riconoscimento viene inficiato dalla presenza di popolazioni avvertite come estranee e capaci di destabilizzare le certezze e le consuetudini, allora la finalità prevalentemente difensiva privilegia l'interesse dei "minacciati" e lascia in ombra la finalità "riadattiva e reintegrativa".

In tal senso, anche $\mathrm{i}$ servizi sanitari $\mathrm{e}$ in particolare quelli psichiatrici sono sollecitati a identificare e a definire l'insorgenza e la presentazione di sintomi, l'andamento clinico, l'outcome, la risposta al trattamento, la facilità $\mathrm{O}$ meno di accesso alle cure. A questo proposito anche la psichiatria e la criminologia psichiatrica avvertono la difficoltà di doversi confrontare con situazioni assai problematiche.

Un esempio emblematico è quello di un giovane albanese, condannato per omicidio volontario e rapina, fatti avvenuti in provincia di Trento nel 2009, il quale, negando qualsiasi responsabiltà, ha sempre affermato di sapere solo che era stato ucciso un ragazzo e di essere estraneo a quell'avvenimento anche se l' unica responsabilità, a suo dire, era quella di essersi trovato "nel momento sbagliato nel posto sbagliato".

Si è potuto constatare che il medesimo è stato in grado di ricostruire molte vicende della propria storia di vita dimostrando in tal modo di non presentare disturbi per quanto riguardava la memoria sia per fatti recenti che fatti lontani, pur in presenza delle consapevoli risposte relative al "non sapere e al non ricordare". Ad esempio, precisò di essersi sposato nel 2007, di aver convissuto sempre con la moglie che incontrava regolarmente tutti $i$ sabati insieme ad altri 
familiari durante i permessi che gli venivano concessi come internato presso l'OPG (Ospedale Psichiatrico Giudiziario) di Reggio Emilia.

Nel corso degli accertamenti psichiatrico-forensi è stato sottolineato che, di fronte alla richiesta di precisazioni o di puntualizzazioni relative al fatto per cui era imputato, ricorreva spesso, come in precedenza sottolineato, alle espressioni "non ricordo", "non sono in grado di precisare".

Questo modo di reagire, collegabile ad una modalità di simulazione, può, tuttavia, per la qualità del suo estrinsecarsi, apparire come un comportamento reattivo di una personalità, caratterizzata da tratti di immaturità, che vive una situazione di grave difficoltà essendo posta di fronte a contingenze eccezionali: il doversi, ad esempio, difendere da un'accusa legata ad un comportamento che egli ha sempre negato. I fattori stressanti che hanno costellato la vita di questo ragazzo, come i dati raccolti nel corso della perizia misero in evidenza, sono la fine di una relazione sentimentale con una fidanzata italiana, il matrimonio "combinato", quindi imposto e solo all'apparenza gradito ed accettato, dal quale nacque una figlia, la perdita di una persona cara e con un ruolo importante, quale è stata la morte dello zio materno considerato dal ragazzo come un padre.

E' evidente che le famiglie straniere vivono e condividono con i nuclei autoctoni molti problemi quotidiani. Tuttavia, esse presentano fragilità specifiche causate proprio dalla migrazione: tra queste merita di essere annoverata l'assenza degli anziani, dei nonni, figure importanti perché depositarie di memorie familiari di cui garantiscono la trasmissione tra le generazioni. E' alla luce di queste considerazioni che può essere letto ed interpretato, nel caso in esame, l'evento stressante della morte dello zio materno che può aver contribuito a creare un "vuoto generazionale"s.

Questi fatti, come evidenziato in precedenza, vanno inseriti in un contesto di personalità scarsamente differenziata ed immatura. L' immaturità della personalità emerge dalla biografia del ragazzo e, in modo significativo, dal test di Rorschach dove il soggetto ha offerto una prova particolarmente povera sia sul piano quantitativo che qualitativo, evidenziando un fondo astenico e depressivo strutturale che determina passività e dipendenza, limitando fortemente le risposte all'ambiente e all'altro.Era altresì presente uno spirito critico ed oppositivo molto marcato, sicuramente compensatorio di un vissuto interno di inadeguatezza, che lo spingeva a porsi in modo critico e polemico nei confronti dell'altro, escludendo qualsiasi atteggiamento di ascolto e di condivisione.

Al test si evidenziava anche un chiaro senso di lontananza dal modo comune di sentire e di pensare che non ha facilitato un adeguato inserimento nel contesto sociale di appartenenza. Sul piano affettivo, la personalità appariva strutturalmente povera ed immatura nelle sue espressioni e la gestione degli impulsi era carente. I meccanismi difensivi erano fragili e la rimozione, tesa ad escludere dalla coscienza determinate rappresentazioni connesse ad una pulsione il cui soddisfacimento entrerebbe in contrasto con altre esigenze psichiche, appariva il meccanismo prevalente.

8 V. Iori, "Famiglie migranti e integrazione scolastica", in E. Scabini, G. Rossi (a cura di), La migrazione come evento familiare, Vita e Pensiero, Milano, 2008. 
Questi tratti di personalità, senz'altro abnormi, possono essere collegati ad un disturbo di adattamento che incide ulteriormente sull'affettività, provocando ansia e depressione del tono dell'umore con compromissione anche dei rapporti interpersonali e delle prestazioni lavorative. Tali situazioni hanno fatto sì che il condannato fosse ritenuto esaurito e malato tanto che i suoi genitori lo portarono all'attenzione del medico di base. Si può pertanto sostenere che, per una serie di difficoltà legate all'ambiente e per le strutture di personalità, questi presentasse prima del fatto per cui fu condannato quei disturbi dell'adattamento che insorgono dopo eventi non accettati e non adeguatamente metabolizzati. Queste considerazioni sollecitano a riflettere sul fatto che anche la globalizzazione dei costumi, dei comportamenti, delle manifestazioni, da alcuni tanto esaltata, anche se soltanto in parte effettiva, vista ed attesa come una panacea universale, destinata a risolvere conflitti e rivalità tra $\mathrm{i}$ popoli e le nazioni, è in realtà molto più complicata di quanto non sembri perché l'identità dell'uomo si costituisce sempre in rapporto con un'alterità.

Infatti, l'identità, o meglio ciò che noi crediamo essere la nostra identità, ciò in cui maggiormente ci identifichiamo, è fatta anche di alterità e costruire l'identità non comporta soltanto un ridurre, un emarginare l'alterità, bensì introdurre ed incorporare l'alterità nei processi formativi dell'identità. L'identità è certamente un principio logico elementare, ma "da sola" è anche fallimentare. L'identità è in effetti un'esigenza irrinunciabile, ma di sola identità si muore ${ }^{9}$.

Da qui il complicato gioco dell'identità che si realizza attraverso l'ambiguità risultante dall"'essere" e dall"'avere". E' difficile separare nel soggetto il suo essere dal suo avere perché il legame tra questi due aspetti è talmente forte che ogni presentazione mobilita, in modo spesso indistinto, l'uno e l'altro aspetto del proprio Sé tanto che, mettendo in discussione uno di questi aspetti, il rischio che si corre è quello di rimettere in discussione pure l'altro.

Nel caso del ragazzo condannato per omicidio, la descrizione è quella di un bambino ed un adolescente sorridente e sereno, per quanto di carattere timido, molto corretto, amante del calcio e della pesca. Cominciò a lavorare presto, a 16 anni, ed arrivò in Italia nel 2002. Fino al 2008 nulla di strano sembra emergere nella sua vita e nel suo comportamento. C'era stata la fine del rapporto sentimentale con una ragazza italiana e vi era stato il matrimonio in Albania, nel 2007, certamente combinato, 'come lo sono tutti i matrimoni', hanno precisato i suoi familiari, perché 'così usa da noi', ma il ragazzo era sembrato felice. Nella seconda metà del 2008, la situazione invece cambia radicalmente sotto l'incalzare di alcuni eventi tragici: muore uno zio, quello a cui il ragazzo era forse più legato. Il ragazzo è disperato, al funerale sviene 'per il dolore'. A dicembre 2008 si registra un altro lutto, muore infatti l'ex datore di lavoro del padre. Qui cominciano ad apparire evidenti stranezze e bizzarrie. Egli inizia a negare la morte dello zio, dice che non è vero, che lo zio è vivo, arriva anche a dubitare di aver fatto un 
incidente poco tempo prima chiedendo conferme ad uno zio, mentre stavano attraversando il luogo dove era avvenuto il sinistro.

Nel caso qui presentato le perizie e le consulenze si sono succedute prendendo in considerazione, nel tentativo di poter frettolosamente etichettare da un punto di vista diagnostico il suo comportamento, la diagnosi di schizofrenia all'esordio e pertanto i suoi gesti, soprattutto quelli caratterizzati da impulsività aggressiva, vennero considerati come sintomatici dell'esordio schizofrenico. Nei suoi confronti venne anche redatta una diagnosi di “episodio psicotico con prevalente contenuto affettivo, ovvero di disturbo schizoaffettivo", trascurando che l'accusa di omicidio e la previsione di una lunga carcerazione ad esso collegata possono configurare un evento straordinario in grado di provocare reazioni di paura e sofferenza in chiunque che può dar luogo a sintomi di tipo ansioso e di tipo dissociativo, con l'irrompere di episodi depressivi.

La biografia e i dati obiettivi emersi dalla documentazione e dalle indagini orientano a leggere il comportamento del soggetto come riconducibile ad uno stato reattivo che non sarebbe insorto senza l'avvenimento-causa. Il soggetto ha sviluppato i sintomi tipici che seguono l'esposizione ad un fattore traumatico estremo, quale può essere considerata la prospettiva di una lunga condanna legata all'accusa di omicidio. D'altro canto il DPTS ( Disturbo Post Traumatico da Stress) è un concetto antico quanto la storia dell'uomo perché fa riferimento al fatto che un violento

9 F. Remotti, Contro l'identità, Laterza, Roma-Bari, trauma psichico, con il significato di grave minaccia e di pericolo perdurante, possa lasciare tracce profonde e talvolta anche indelebili nella mente umana.

Di conseguenza, prendere in esame contestualmente colloquio, test di Rorschach e storia di vita è un approccio che può produrre validi risultati nell'ambito criminologicopsichiatrico $^{10}$. Infatti, nella prospettiva della perizia psichiatrica e delle altre indagini consentite, ad esempio, si può sostenere che l'utilizzazione del DSM o dell'ICD-10 è possibile a condizione che si operi con attenzione attraverso la storia di vita (o biografia clinica del soggetto). In questa situazione, il metodo biografico, nei tre momenti essenziali (di raccolta, di ordinamento e di esposizione del materiale) diventa fondamentale per la prognosi e trova nella valutazione diagnostica multiassiale una possibilità di dare conferma alla descrizione. Affrontare il tema della salute mentale degli immigrati, ad esempio, a partire dalle diverse società di provenienza significa inevitabilmente chiamare in causa l'antropologia culturale, significa altresì considerare la salute e la pratica delle cure non unicamente come tecniche sviluppate in un campo scientifico postulato come universale ma, in presenza di una realtà nuova e diversa, significa integrare i sistemi culturali delle minoranze allogene, che hanno sviluppato teorie dell'uomo e del suo posto

1996, pag. 57.

10 R. Bisi (a cura di), Psicodiagnostica e storie di vita in criminologia. Un'analisi attraverso l'omicidio, Franco Angeli, Milano, 2004; A. Balloni, "Psichiatria forense", in G. B. Cassano, P. Pancheri et al. (a cura di), Trattato italiano di psichiatria, Masson, Milano, 1999, pp. 3899-3916; R.Bisi, "La psicodiagnostica in criminologia: orientamenti e prospettive", in G. Mucciarelli, R.Chattat, G.Celani (a cura di), Teoria e pratica dei test, Piccin, Padova, 2002, pp. 508-527. 
nell'universo, in funzione delle credenze e delle conoscenze che sono state elaborate sul tema del mantenimento della salute.

In altri termini, nel momento in cui la cura diviene necessaria nelle società umane eterogenee e allorquando il paziente è inserito in un contesto di riferimento diverso da quello del medico, se si vuole che l'intervento sia efficace è sempre più necessario domandarsi che senso esso rivesta per $i$ protagonisti di questa situazione interculturale.

Le condizioni psicopatologiche alle quali sono sottoposti i protagonisti di simili situazioni è ben esemplificata dall'esperienza clinica riportata da un dirigente psicologo dell'Unità di Psichiatria dell'ospedale di Agrigento ${ }^{11}$.

Alcuni anni fa venne ricoverato presso l'Unità di Psichiatria dell'ospedale di Agrigento, in Sicilia, un giovane di cui risultò estremamente difficoltoso raccogliere dati anagrafici, clinici e notizie sul suo contesto di vita. Di certo, evidenziava in maniera esponenziale $\mathrm{e}$ amplificata i problemi e gli aspetti psicologici di un giovane migrante.

Era sbarcato a Lampedusa da uno dei famosi barconi della "speranza". Le notizie anamnestiche di questo giovane apparvero, fin da subito, scarne e povere di informazioni. Egli era un giovane eritreo di 24 anni, celibe, che, giunto sullisola, fu urgentemente ricoverato presso l'ospedale di Agrigento per malnutrizione, dimagrimento e attacchi di panico. Fu chiesta anche la consulenza degli operatori dell'Unità di psichiatria perché il giovane evidenziava restringimento del campo di coscienza e un

11 F. Sciacca, "Evento migratorio e reazione psicogena acuta", Babele, a. VIII - n. 35, gennaioaprile 2007 , pp. 47-51. comportamento bizzarro caratterizzato da immobilità o da movimenti afinalistici. Inoltre non parlava e perciò fu considerato sordomuto, o disfonico (infatti fu richiesta una visita otorino). Si stava così creando l'idea, la sensazione, l'immagine che il giovane fosse un paziente irrecuperabile.

Vista la difficile gestione del paziente, egli fu trasferito all'Unità di Psichiatria dell'ospedale di Agrigento dove rimase per 45 giorni. Fu chiesta l'attivazione dei servizi sociali per stabilire il luogo che lo avrebbe ospitato dopo le dimissioni perché considerato rifugiato politico.

A partire dalle scarne notizie si ricostruirà che il motivo della migrazione del giovane era dovuta al fatto di essere stato renitente al servizio militare eritreo, di essere fuggito per evitare la guerra come altri giovani eritrei suoi coetanei. Vista la difficile condizione psico-fisica e soprattutto in considerazione del fatto che non parlava, furono inizialmente osservati con attenzione i segni clinici e i suoi comportamenti. Evidente era il dimagrimento, la disidratazione, il blocco psicomotorio (stava per ore sdraiato per terra o fermo in una posizione). L'espressione del volto era perplessa, sofferente e triste.

Il quadro clinico indicava pertanto una reazione psicogena acuta da stress grave, determinata dallo choc subito dall'evento migratorio (e quindi choc culturale) con grave rallentamento psicomotorio e mimico-gestuale, scarso contatto visivo, mutismo senza diretta risposta agli stimoli, catatonismo con assunzione di posture bizzarre, compiva azioni motorie di significato opposto e mostrava resistenza alle istruzioni.

La psicologia culturale e la letteratura transculturale individuano l'incidenza dei problemi della migrazione principalmente nei 
traumi, nello choc culturale, nei vissuti di sradicamento e di distacco dalla famiglia e dal mondo degli affetti. La migrazione è un cambiamento così profondo che può produrre molta sofferenza sulla psiche della persona, sul suo funzionamento, tanto più se la migrazione è stata forzata.

La migrazione allora diviene un trauma che genera stress psichico, sentimenti di impotenza, perdita dell'autostima, emozioni intense e spesso congelate che emergono, spesso dissociate dalla parola, sotto forma di sensazioni somatiche e reazioni comportamentali.

La migrazione rappresenta un'esperienza traumatica e di crisi che, per quanto concerne la difficoltà e al contempo l'estraneità del migrante, può rimandare al quadro "Piazza d'Italia" di de Chirico che esemplifica molto bene, a mio avviso, l'ambiguità del richiamo esercitato dalle nostre città: da un lato, la costruzione evoca quella delle "citta ideali" rinascimentali dalla prospettiva perfetta, tuttavia, la posizione centrale, occupata dalla statua di Arianna, le due figure umane e, sullo sfondo, in lontananza, il treno in movimento ci suggeriscono una sostanziale e inesorabile estranetà dell'elemento umano ad una prospettiva così lucida e perfetta.

Con riferimento al caso del giovane eritreo, occorre precisare che gli operatori del reparto, come riporta nell'articolo il Dirigente psicologo dell'Unità di Psichiatria di Agrigento, hanno cercato di stabilire con il giovane un minimo contatto, anche attraverso il non verbale, al fine di renderlo più collaborativo. Successivamente sono stati invitati come mediatori due connazionali eritrei che parlavano la stessa lingua: anche con loro il giovane non sembrava mostrare ascolto e non manifestava feedback alle loro domande e ai loro discorsi. Si rinforzava in tutti gli operatori, pertanto, l'idea che il giovane fosse sordomuto. Venne somministrata anche una terapia psicofarmacologica. Tuttavia, gli operatori hanno mantenuto con il giovane un atteggiamento di accoglienza, di pazienza, ma al contempo di stimolo attraverso la gestualità, lo sguardo e il sorriso. Venne ovviamente utilizzata in modo massiccio la comunicazione non verbale. Gradualmente egli cominciò ad essere più collaborativo (ad esempio, seguiva lo psicologo mentre camminava, entrava nella sua stanza, si accomodava).

La progressiva e maggiore disponibilità a collaborare del giovane ha consentito allo psicologo di fare alcuni iniziali tentativi di interazione con l'uso della penna, della matita e dei fogli. Usando la scrittura, gli operatori gli rivolsero domande in lingua inglese per verificare se la conoscesse e, con sorpresa, constatarono che, a stento, il giovane iniziava a scrivere in inglese. Solo molto tempo dopo fu anche possibile farlo disegnare (disegno della casa, dell'albero).

Tramite questo intervento fu possibile raccogliere le notizie anamnestiche mancanti. Innanzitutto, egli non sapeva di trovarsi in Italia, scrisse la sua età, si seppe che aveva tre fratelli e due sorelle, che gli piaceva studiare e che le sue materie preferite erano la chimica e la matematica.

Dal modo in cui forniva informazioni e dai contenuti espressi si poté constatare che le sue funzioni cognitive apparivano integre. Ringraziò $i$ medici, affermando che non avrebbe mai dimenticato il loro aiuto. Progressivamente egli cominciò ad usare la voce, parlando prima in inglese e poi, con i mediatori, nella sua lingua. 
Ovviamente gli operatori cominciarono a modificare l'idea, la sensazione e l'immagine di non recuperabilità che si era costruita intorno al giovane. Dopo la dimissione è stato in grado di raggiungere i suoi amici di Genova, in precedenza contattati dai servizi sociali, che si resero disponibili ad ospitarlo.

L'illustrazione di questo caso permette un'ulteriore considerazione sulla difficoltà che il giovane aveva a rappresentarsi e a descrivere la propria vita psichica e la tendenza a esperire e a comunicare la sofferenza nella forma di sintomi somatici e a parlarne soltanto in tal senso. Era come se questo giovane non sapesse esprimere e definire con chiarezza i contenuti psichici; il suo vissuto non era messo a fuoco e riconosciuto, ma rimaneva vago e nebuloso. Le manifestazioni della sua vita psichica non potevano altrimenti essere espresse e spiegate se non attraverso il filtro del corpo e la sofferenza somatica.

E' noto che tale difficoltà nel riconoscere ed esprimere verbalmente la sofferenza interna é segno caratteristico dell'alessitimia che significa letteralmente "affetto senza parole" (dal greco $a$ lexis (discorso, parola) thymòs (affetto, emozione) e definisce propriamente l'incapacità di comunicare verbalmente le proprie emozioni. La menomazione della capacità di utilizzare le emozioni come segnali da parte dei pazienti alessitimici è basata sulla forma che assumono le loro risposte emotive. Le loro reazioni "sono fondamentalmente somatiche e consistono degli aspetti 'espressivi', o fisiologici, degli affetti con una verbalizzazione minima [...]. Spesso gli alessitimici non riescono a dire se sono tristi, stanchi, affamati o malati. Non sono abituati a riconoscere i propri stati sentimentali e a rendersi conto delle proprie reazioni agli eventi della vita"12.

Gli studi transculturali evidenziano le profonde differenze che esistono nell'esperienza e nell'espressione degli affetti, soprattutto nell'espressione corporea della sofferenza, che minimizza le componenti psichiche ed emotive. Già nel 1963 gli studi degli psicoanalisti francesi Marty e de M'Uzan'13 avevano sottolineato l'importanza dell'uso del concetto di "pensée operatoire" per designare un tipo di funzionamento mentale che implica una concentrazione sulle cose a scapito dei rapporti oggettuali.

E' evidente che i mutamenti repentini e frequenti propri della nostra società, in parte provocati anche dai flussi migratori, rendono più difficile la possibilità di mettere a frutto quanto si è appreso in passato al fine di mantenere il senso della continuità nel tempo.

Affrontare i problemi posti dai flussi migratori significa altresì fare i conti con il rischio, reagire all'ingiustizia, nutrire la propria coscienza di nuovi significati e di nuovi valori poiché queste sono condizioni che caratterizzano il tempo che viviamo, contraddistinto da timori vecchi e nuovi che mettono a repentaglio la nostra quotidianità, ponendoci di fronte a grandi ed importanti sfide.

D'altro canto tutti gli incontri così come tutti gli eventi non preordinati scombussolano l'ordine predefinito, asettico, dove tutto scorre seguendo un suo corso ed allora vorrei rifarmi ad un

\footnotetext{
${ }^{12}$ H. Krystal , Affetto, trauma, alessitimia, Edizioni Magi, Roma, 2007, pag. 308.

${ }^{13}$ P. Marty , M. de M'Uzan , "La pensée operatoire “, Revue Française de Psychanalyse, 27, pp. 345-356, 1963.
} 
recente libro ${ }^{14}$ di E. Ferrero con contenuto similfiabesco, declinato in chiave metaforica. Nel libro si parla di Quirina, un'anziana signora che vive in orgogliosa solitudine in un piccolo paese delle Alpi, dove accudisce un orto-giardino che è tutta la sua gioia, uno spazio armonioso che vuole opporsi all'insensato disordine del mondo. Finché una mattina di maggio fa una scoperta che la sconvolge: il prato è rovinato da una serie di mucchi di terra che rivelano la presenza di una talpa. Comincia una guerra senza quartiere nei confronti di questa "intrusa", scomoda e fastidiosa inizialmente, in cui, al fine di combatterla, vecchie soluzioni e credenze popolari si alternano con le nuove tecnologie. L'intrusa, tuttavia, scatena interrogativi e inquietudini, evoca memorie che sembravano perdute e, dopo avere combattuto vanamente disordine e caos, Quirina si rende conto di non poter vivere senza l'intrusa che, sebbene abbia spezzato equilibrio ed armonia, ha rivitalizzato e conferito senso e direzione ad una quotidianità ripetiva e monotona.

\section{Bibliografia di riferimento.}

- Altobelli D., "I luoghi della tipicità e della tradizione", in Ruini M., (a cura di), Dentro e fuori la città, Edizioni Nuova cultura, Roma, 2010.

- Balloni, "Psichiatria forense", in Cassano G. B., Pancheri P. et al. (a cura di), Trattato italiano di psichiatria, Masson, Milano, 1999.

- Beneduce R., Frontiere dellidentità e della memoria, FrancoAngeli, Milano, 1988.

- Bisi R., "La psicodiagnostica in criminologia: orientamenti e prospettive", in Mucciarelli G., Chattat R., Celani G. (a cura di), Teoria e pratica dei test, Piccin, Padova, 2002.
- $\quad$ Bisi R. (a cura di), Psicodiagnostica e storie di vita in criminologia. Un'analisi attraverso l'omicidio, Franco Angeli, Milano, 2004.

- Bisi R., "Quale spazio per la vittima nella società contemporanea?", in Balloni A. (a cura di), Cittadinanza responsabile e tutela della vittima, Clueb, Bologna, 2006.

- Ceccanti S., "Se l'Italia si 'difende' dal burqa e dimentica la cittadinanza”, Reset, 134,2012 (disponibile al sito: http://www.reset.it/magazine/134).

- de Martino E., La fine del mondo. Contributo all'analisi delle apocalissi culturali, Einaudi, Torino, 1977.

- Ferrero E., Storia di Quirina, di una talpa e di un orto di montagna, Einaudi, Torino, 2014.

- Iori V., "Famiglie migranti e integrazione scolastica", in Scabini E., Rossi G. (a cura di), La migrazione come evento familiare, Vita e Pensiero, Milano, 2008.

- Krystal H., Affetto, trauma, alessitimia, Edizioni Magi, Roma, 2007.

- Liotta E., Su anima e terra. Il valore psichico del luogo, Magi, Roma, 2005.

- Marty P., de M'Uzan M., "La pensée operatoire ", Revue Française de Psychanalyse, 27, pp. 345-356, 1963.

- Nussbaum M., "Proibire il velo significa discriminare", Reset, 134, 2012 (disponibile al sito: http://www.reset.it/magazine/134).

- Parolari P., "Reati culturalmente motivati.Una nuova sfida del multiculturalismo ai diritti fondamentali”, Ragion pratica, 31, 2008, pp. 529-558.

- Remotti F., Contro l'identità, Laterza, RomaBari, 1996.

- Sciacca F., "Evento migratorio e reazione psicogena acuta", Babele, a. VIII, n. 35, gennaio-aprile 2007, pp. 47-51.

- Welchman L., Honour and Violence Against Women in a Modern Shar'I Discourse (disponibile al sito: http://eprints.soas.ac.uk/7665/1/Welchma n in HAWWA 2007 pre-publication.pdf).

\footnotetext{
${ }^{14}$ E. Ferrero, Storia di Quirina, di una talpa e di un orto di montagna, Einaudi, Torino, 2014.
} 